\title{
Mean fecal glucocorticoid metabolites are associated with vigilance, whereas immediate cortisol levels better reflect acute anti-predator responses in meerkats
}

\author{
Irene K. Voellmy ${ }^{1,5,6}$, Ines Braga Goncalves ${ }^{1,5}$, Marie-France Barrette ${ }^{2,3,5}$, Steven L. \\ Monfort $^{3}$, Marta B. Manser ${ }^{1,4,5}$
}

${ }^{1}$ Institute of Evolutionary Biology and Environmental Studies, University of Zurich, Winterthurerstrasse 190, 8057 Zurich, Switzerland

${ }^{2}$ Département de Biologie, Université de Sherbrooke, Sherbrooke, J1K 2R1 Canada

${ }^{3}$ Smithsonian Conservation Biology Institute, National Zoological Park, 1500 Remount Rd, Front Royal, VA, 22630 USA

${ }^{4}$ Mammal Research Institute, Pretoria University, 00002 Pretoria, SA

${ }^{5}$ Kalahari Meerkat Project, Kuruman River Reserve, South Africa

${ }^{6}$ Present address: School of Biological Sciences, University of Bristol, Life Sciences Building, 24 Tyndall Avenue, BS8 1TQ, Bristol, UK

Corresponding author:

Marta B. Manser, Institute of Evolutionary Biology and Environmental Studies, University of Zurich, Winterthurerstrasse 190, 8057 Zurich, Switzerland, marta.manser@ieu.uzh.ch

\section{Abstract}

Adrenal hormones likely affect anti-predator behavior in animals. With experimental field studies, we first investigated associations between mean fecal glucocorticoid metabolite (fGC) excretion and vigilance and with behavioral responses to alarm call playbacks in free-ranging meerkats (Suricata suricatta). We then tested how vigilance and behavioral responses to alarm call playbacks were affected in individuals administered exogenous cortisol. We found a positive association between mean fGC concentrations and vigilance behavior, but no relationship with 
the intensity of behavioral responses to alarm calls. However, in response to alarm call playbacks, individuals administered cortisol took slightly longer to resume foraging than control individuals treated with saline solution. Vigilance behavior, which occurs in the presence and absence of dangerous stimuli, serves to detect and avoid potential dangers, whereas responses to alarm calls serve to avoid immediate predation. Our data show that mean $\mathrm{fGC}$ excretion in meerkats was associated with vigilance, as a re-occurring anti-predator behavior over long time periods, and experimentally induced elevations of plasma cortisol affected the response to immediate threats. Together, our results indicate an association between the two types of anti-predator behavior and glucocorticoids, but that the underlying mechanisms may differ. Our study emphasizes the need to consider appropriate measures of adrenal activity specific to different contexts when assessing links between stress physiology and different anti-predator behaviors.

Keywords: adrenal activity; alarm calls; anti-predator behavior; communication; cortisol; fecal glucocorticoids; meerkats; Suricata suricatta; vigilance

\section{Introduction}

Adaptive behavioral responses to environmental challenges are crucial to secure individual survival and reproductive success (Boonstra, 2005; McEwen and Wingfield, 2003). Adrenal hormones have important metabolic, cardiovascular, immunologic and homeostatic functions (e.g. Harvey et al., 1984, Bush and Hayward, 2009, Zanchi et al., 2010, Goymann 2012), which in turn, can affect individuals' response to internal and external stimuli (Dowle et al., 2012). Under challenging conditions, such as food deprivation, social instability or predation risk (Dallman et 
al., 1993; Creel et al., 2009, 2013) adrenal activity increases the production of glucocorticoids (GCs) to mobilize energy and resolve stressful stimuli (Harvey et al., 1984; McEwen and Wingfield, 2003; Wingfield, 2005; Romero et al., 2009). In turn, GCs can modulate the intensity of behavioral responses to stimuli such as presence of predators (Sapolsky et al., 2000). For instance, in tree lizards (Thaker et al., 2009) and in rats (Rosen et al., 2008) administration of exogenous GCs elicited stronger responses to predator cues. Thus, the commonly observed individual variation in anti-predator behavior could be influenced by variation in adrenal activity with, for instance, individuals with chronic elevations in GCs responding more intensely to potential threats (Bercovitch et al., 1995; Blumstein et al., 2006; Blumstein and Chi, 2012).

Predation risk, body condition, and social context affect anti-predator behavior including vigilance, and response to alarm calls. Vigilance behavior that enhances the detection of threats and serves to avoid predation (Hunter and Skinner, 1998) increases with predation risk (e.g. Welp et al., 2004, Clutton-Brock et al., 1999a, Mateo, 2007), satiation and body condition (Bachman 1993, Clutton-Brock et al., 1999b), and can also be affected by the social environment an individual experiences. For example, in many gregarious bird and mammal species, individual vigilance levels are negatively correlated with group size (reviewed in Caro 2005, Lima and Dill, 1990) and, in meerkats, individuals increase vigilance rates in the presence of dependent young (Santema and Clutton-Brock 2013). Since all these factors can increase fGC excretion (Goymann, 2012), these behavioral responses are likely associated with adrenal activity (Blumstein et al., 2006), as has been shown for individual variation in meerkat sentinel behavior (Tatalovic, 2008; Santema et al. 
2013) and in Belding's ground squirrels' response to alarm call playbacks (Mateo, 2007).

Natural variation in adrenal activity can be assessed directly by sampling plasma cortisol levels (Romero and Reed, 2005) or indirectly by measuring GCs excreted in feces (Wasser et al., 2000; Whitten et al., 1998). Plasma samples yield a single "point in time" measure informative of an individual's immediate endocrine status, with however the potentially confounding effects of handling, capture and anesthesia (Sheriff et al., 2011). In contrast, feces offer the opportunity for repeated non-invasive sampling of free-ranging animals, thus providing integrated measures of biologically active GCs excreted over longer periods (Sheriff et al., 2010), from hours to days, depending on species-specific excretion rates (Palme et al., 2005). Despite high intra-individual variation in $\mathrm{fGC}$ measures, which reduce our ability to detect internal and external factors associated with adrenal activity (Palme et al., 2005, Touma and Palme 2005 and Goymann 2012), averaging multiple samples from the same individual over long periods of time could provide unique information regarding interindividual differences in general hormonal state.

In meerkats, Suricata suricatta, anti-predator behavior has been extensively studied (e.g. Clutton-Brock et al., 1999a, 1999b; Manser, 2001) and methods to assess adrenal activity in feces are well established (Young et al., 2006). Meerkats are small, cooperatively breeding mammals living in groups of 3 to 50 individuals (Clutton-Brock et al. 2006) in arid, open habitat areas of Southern Africa. They dig for invertebrates and small vertebrates in sandy substrates (Doolan and Macdonald, 1996), which prevents them from watching for predators while foraging (CluttonBrock et al., 1999b). In response to high predation risk, meerkats have evolved anti- 
predator behavior using sentinels (Clutton-Brock et al., 1999b; Manser 1999) and an acoustic communication system of alarm calls that differ depending on type of predator and level of urgency (Manser, 2001; Manser et al., 2002). Previous studies have shown that meerkats exhibit high individual variation in vigilance behavior (Clutton-Brock et al., 1999a), and in intensity of their behavioral response to alarm calls (Amsler, 2009). Sentinel behavior in meerkats is positively associated with fGC concentrations (Tatalovic, 2008; Santema et al. 2013). In addition, dominance status (Carlson et al., 2004) and the presence of pups are associated with higher plasma cortisol concentrations (Carlson et al., 2006). Hence, the observed variation in vigilance behavior during foraging and in response to predator alarms may also be modulated by individual adrenal activity.

In this study, we first quantified mean fGC concentrations in meerkats displaying two different categories of anti-predator behavior: vigilance during foraging, and response to alarm calls. Secondly, we compared individual response intensity and alertness before and after playbacks of alarm calls in individuals administered either exogenous cortisol or saline solution, as control. We predicted that elevated mean fGC concentrations should be associated with increased vigilance behavior while foraging and with stronger response to alarm call playbacks, i.e. eliciting a stronger first response and increased latencies to resume foraging after the playback. We also expected exogenous cortisol administration to increase response intensity to the alarm call playbacks. To our knowledge, this is the first study to investigate the relationship between glucocorticoids and two different types of anti-predator behavior in a population of free-ranging animals. 


\section{Methods}

(a) Study site and animals

Our study was conducted at the Kuruman River Reserve in South Africa during the meerkats' breeding season, from November 2007 to January 2008 and from October 2011 to March 2012. The study site (Kalahari Meerkat Project) is located $30 \mathrm{~km}$ west of Van Zylsrus in the southern part of the Kalahari desert $26^{\circ} 58^{\prime} \mathrm{S}, 2^{\circ} 49^{\prime} \mathrm{E}$ (CluttonBrock et al., 1998a). In 2007-08, we worked with six habituated meerkat groups consisting of six to 11 individuals. In 2011-12, we performed 23 playback trials on pairs of individuals in eight groups consisting of 11 to 34 individuals. Long-term behavioral data and life histories were known for all individuals. The animals were individually tagged with subcutaneous transponder chips and black hair-dye marks were frequently renewed for visual identification (Jordan et al., 2007). All animals were habituated to a level that made observations possible within $1 \mathrm{~m}$. Thereby, observers remained at a distance that would not induce any disturbance, i.e., visible behavioral changes in the meerkats such as interruption of foraging to look at the observer.

To control for the potential confounding effects of age, dominance status and reproductive state on adrenal activity (Carlson et al., 2004, Barrette et al., 2012) we included only adult subordinate individuals aged between 1 and 2.5 years, in our study. Juveniles were excluded from the present study because they respond to threats differently compared with adults, due to their earlier developmental stage (Hollén and Manser, 2006). 
(b) Data collection

\section{i) Vigilance}

Vigilance behavior was assessed from November 2007 to January 2008 by IKV using behavioral group scans (Altmann, 1974) in six groups during three or four mornings within one month, while the animals were foraging. Vigilance behavior was defined as head lifting and scanning the environment, while standing quadrupedally, or while standing or sitting on hind legs. Occurrence of any vigilance behavior by any adult subordinate individual was noted every 15 min over two to three hours until the animals began resting during the hot midday time. The total number of scans completed at the end of each observation day was used to calculate numbers of times an animal was observed non-vigilant for subsequent data analysis.

\section{(ii) Alarm call playback experiments}

Experiment I - natural response to alarm calls

With playback experiments, we tested the behavioral response to alarm calls in the absence of predators using recordings of six low urgency and six medium urgency aerial alarm calls (Manser, 2001; Manser et al., 2002) from the long-term audio recording database (Manser, 1995 to 2007). Each alarm call had been recorded from adult meerkats of different groups unfamiliar to the focal individuals (Manser et al., 2001; Schibler \& Manser, 2007). All alarm call files were edited in Cool Edit 2000 (Syntrillium Software Corporation, Phoenix, AZ, USA). Edited sound files were transferred to a Marantz PMD-660 solid state audio recorder (Marantz Japan Inc.), and played through a walkman loudspeaker (JBL, on tour, Los Angeles, USA, frequency response $100-20^{\prime} 000 \mathrm{~Hz}$ ). Amplitude of both call types were adjusted to naturally observed volumes of $56-60 \mathrm{~dB}$, measured 1 meter away from the speaker (Cirrus CR: 261 sound level meter, Cirrus Research plc., Hunmanby, UK). All 
playback experiments were conducted while the animals were foraging and no natural alarm calls had occurred during the preceding 10 minutes. The speaker was positioned 10 to 15 meters away from the focal animal and at least 10 meters away from any other group member. To avoid motivational biases, playbacks were directed at animals that were searching for prey, but not engaged in focused digging (Amsler, 2009). During each experimental session we conducted a maximum of two playbacks, with a minimum time interval of 45 minutes in between. Within a session, each call type was used only once, and the sequence of call types (low and medium urgency) was randomly chosen to avoid order effects. Playback sessions within the same group were at least four days apart. A total of nine low urgency and 15 medium urgency aerial alarm call playback experiments were conducted in six groups during the afternoons, between 16:00 and 19:00 hours, while the groups were foraging and before they returned to their sleeping burrows. Not all focal individuals were tested with both call types because they were absent from their group on the day of experiments, either due to babysitting (Clutton-Brock et al., 1998a), eviction (CluttonBrock et al., 1998b), or roving (Young et al., 2007); or because preconditions for the playbacks were not met. Each group was tested at least once for each urgency level, but no more than four times. The response of the focal animal was filmed using a Panasonic digital video camera (NV-GS150EG).

Videotapes were digitized for video analysis using Windows Movie Maker 5.1. From the videos, three behavioral responses were measured (by IKV) to quantify each individual's reaction to the playbacks. i) Immediate response: the first behavior shown after the first alarm call within the playback, was recorded as one of seven response categories from least to most extreme intensity: 1) no response; 2) looking up standing on four legs; 3) looking up standing on four legs and vocalizing; 4) 
standing or sitting on hind legs and scanning the environment; 5) standing or sitting on hind legs, scanning the environment and vocalizing; 6) running; and 7) running towards shelter. First response to low urgency alarm calls could only be determined in eight of nine individuals because long grass obscured the view. (ii) Latency until first foraging: time elapsed (in s) from the first response to resume first foraging behavior. iii) Latency until resumption of uninterrupted foraging: time elapsed from the first response to uninterrupted resumption of normal foraging for at least $20 \mathrm{~s}$. Response duration for ii) and iii) were measured using frame-by-frame measurements and a time resolution of $0.08 \mathrm{~s}$.

\section{Experiment II - effects of cortisol administration}

In each playback trial two meerkats, matched for age, sex and rank (usually littermates and never more than 6 months difference in age), were injected either with $0.05 \mathrm{ml}$ saline solution (control treatment) or with $10 \mu \mathrm{g}$ hydrocortisone 21 hemisuccinate sodium salt, (Sigma Aldrich, Buchs, Switzerland) dissolved in $0.05 \mathrm{ml}$ saline solution (cortisol treatment). This procedure causes temporary elevation of plasma cortisol levels (within their natural range, Barrette et al., 2012) and reduction in foraging behavior over at least 2 hours following a single application (Satema et al., 2013). Intra-muscular injections were given in the thighs using a $1 \mathrm{ml}$ syringe with a 25G needle, while the meerkats were sunning, unrestrained, after emerging from their sleeping burrows and before they began foraging (for more details see Santema et al., 2013). Most animals did not show an obvious response to the injections, and the few that did, only showed mild responses moving a short distance away from the experimenter, and resumed sunning within a few seconds. All trials were conducted when pups were foraging with the groups. The effect of exogenous cortisol or saline administration on individual response to alarm call playbacks was assessed using 
recordings of six different medium-urgency aerial alarm calls from the long-term recording database (Manser, 1995 to 2007). Twenty-three trials were performed. However, due to unfavorable weather conditions and technical difficulties, we were only able to analyze eighteen trials conducted in eight groups.

Playback trials were performed after the group had been foraging for most of the morning period; about $2 \mathrm{~h}$ after the individuals received their injections, and no natural alarm calls had occurred in the preceding 10 min. Edited playback calls were uploaded to a Marantz PMD-670 solid state audio recorder and played through a loudspeaker (iHome, model: iHM79, SDI Technologies Inc. USA). The calls were played at waist height to simulate a meerkat on sentinel duty on a tree trunk, at equal distances (between 10 and $15 \mathrm{~m}$ ) from the two focal animals. Two observers, blind to the treatments, followed and filmed (with a Sanyo digital camera FH1 and a SONY handycam HDR-CX130) the focals. Their behavior was filmed starting $30 \mathrm{~s}$ before the alarm call was played back and continued until the focal resumed uninterrupted foraging for at least $20 \mathrm{~s}$ after the playback. Playback sessions within the same group were set at least seven days apart. All videos were analyzed by IBG, blind to the treatments, using The Observer (NOLDUS Observer XT 7.0, Noldus Information Technology, Netherlands). The magnitude of the focals' behavioral response to the playbacks was classified using two different reactions: first response, i.e., the immediate behavior shown by the focal upon hearing the playback call; and the strongest response to the playback calls, using the same scale as for the first playback experiment although adding an $8^{\text {th }}$ category: go into a bolthole or sleeping burrow. In addition, latency until first foraging behavior and latency until resumption of uninterrupted foraging, for at least $20 \mathrm{~s}$, were recorded. 
(iii) Fecal sample collection and hormonal analyses

Fecal samples were collected opportunistically, from November 2007 to January 2008. To test for associations of $\mathrm{fGC}$ concentrations with vigilance rates and with responses to alarm call playbacks, all fecal samples collected from an individual within one week before and after vigilance behavior was recorded and all fecal samples collected within two weeks before and after playback experiment I were used to calculate mean fGC concentrations for subsequent analysis. Samples were collected after the focal animals had moved away from the area to avoid disturbing the animals with the procedure. Samples were immediately placed on ice in thermos flasks, stored within four $\mathrm{h}$ at $-20{ }^{\circ} \mathrm{C}$ and shipped frozen to the Smithsonian Conservation Biology Institute in Front Royal, USA. For the playbacks conducted after administration of cortisol and saline control, between October 2011 and March 2012, no fecal samples were collected.

Concentrations of $\mathrm{fGC}$ metabolites were quantified in a single assay using a doubleantibody ${ }^{125}$ I RIA for corticosterone (ICN Biomedicals, Inc., Costa Mesa, DA), and previously described extraction and radioimmunoassay procedures (Monfort et al., 1997; Young et al., 2006). Analysis of GCs metabolite was preferred over epinephrine, as a physiological measure of stress response, because they are relatively stable, enabling their measurement in feces and their administration to animals under field conditions. Furthermore, previous studies have successfully elevated circulating GCs in meerkats using the same methodology as in the current study (Santema et al. 2013) and the measurement of GC metabolite concentrations in meerkat feces has been validated (Young et al., 2006). Samples were assayed within nine months of collection. Assay sensitivity was $25 \mathrm{ng} / \mathrm{ml}$ and intra-assay variation was $<10 \%$. 


\section{(iv) Statistical analysis}

Proportional and numerical response variables were analyzed in $R$ version 2.15 .2 ( $R$ Core Team, 2012), using mixed models to account for repeated measures of the same meerkat group, individual and treatment pair. For ordinal response variables non-parametric tests were used (first and strongest response to alarm call playbacks), which were analyzed in SPSS 16.0/19.0. Significance was set at $p \leq$ 0.05, and all tests were two-tailed. Mean fGC metabolite concentrations were calculated for each individual, ( $n=2$ to 4 samples per individual), irrespective of time of day of collection, as we found no effect of time of collection (AM/PM) on fCG concentrations (exact Wilcoxon matched-pairs signed ranks test: $n=17, Z=-1.254$, $p=0.225)$. Inclusion of individuals of which only two fecal samples were collected did not impact on results; hence the maximum available sample size was used $(n=18$ for vigilance rates, $\mathrm{n}=16$ for playback experiment I). To assess associations between mean fGC concentrations and vigilance rates, a generalized linear mixed model (GLMM) with binomial error distribution and logit link was used including mean fGC concentrations as independent variable and vigilance rates as proportional response variable, using the 'cbind' function with number of times an individual was observed vigilant and number of times an individual was observed non-vigilant. The GLMM was conducted using the Imer function in the 'Ime4' package (Bates et al. 2012). The full model was compared to a model containing the random group factor only using an ANOVA Chi-squared-test to examine effects of fGC concentrations on vigilance rates.

Latency to first foraging behavior and latency to forage without interruption for at least $20 \mathrm{~s}$ in response to aerial alarm call playbacks were modeled, separately, as response variables in mixed model Cox proportional hazards regressions 
(MMCoxPH) using 'coxme' function, 'coxme' package (Therneau, 2012); fitted by maximum likelihood. To assess the effects of fGC concentrations, full models contained fGC concentrations as independent continuous variable, playback treatment (low urgency, medium urgency aerial alarm calls) as fixed factor and the interaction between the two. To account for partial repeated testing, as some animals (8 of 16) were tested on both call types, subject ID was included, nested within group ID, as a random factor. 'Sex' was not included in the models because a high number of females had been evicted from their natal groups at the time the experiments were conducted, resulting in highly skewed male/female ratios. Age was also not included; instead, data variance was kept to a minimum by only including animals aged between 1 and 2.5 years. To assess the effect of exogenous cortisol administration, full models contained treatment (cortisol, saline control administration), sex and their interaction as fixed factors. To account for pair-wise cortisol/saline administration, treatment dyads were included as a random factor nested in group ID. Stepwise backward model simplification was used to determine the minimal model, with significance of model terms assessed by change in deviance upon removal of terms (ANOVA model comparison, Chi-squared test). For all analyses, odds ratios with 95\% confidence intervals $(\mathrm{Cl})$ of fixed effects for the minimal model were calculated using 'fixef' function in the 'Ime4' package (Bates et al., 2012).

First response intensity in both playback studies was assessed with non-parametric statistics. For the first playback study, we calculated Kendall's tau b correlation matrices to test whether mean $\mathrm{fGC}$ concentrations were associated with the intensity of the first response to low and medium urgency alarm calls. Matrices were inspected for congruent correlation patterns to verify the reliability of significant correlation probabilities. Kendall's tau b correlation was preferred over Spearman's rho 
correlation, as its coefficient calculates the proportion of concordance between two columns of ranked data, instead of calculating differences between ranks of data pairs, as in Spearman's rank correlation. Thus, Kendall's tau b correlations are less sensitive to outliers than Spearman's rank correlations, a particularly important factor when analyzing small sample sizes (Arndt et al., 2000; Lapata, 2006). The effect of cortisol and saline treatments on the intensity of the first response and the strongest response to medium-urgency alarm calls was assessed using Wilcoxon exact tests. Effect sizes for Wilcoxon exact tests were calculated using the formula: $r=Z / \sqrt{ } n$ ( $n=$ total number of observations $=36$, according to Field, 2005).

\section{Results}

Mean fGC concentrations in meerkats showed an association with individual vigilance rate, but not with response intensity to alarm call playbacks. Individuals spent between 0 and $63.6 \%$ of their time vigilant (mean $\pm s e=26.2 \pm 2.4 \% ; n=18$ individuals in 6 groups), and mean fGC concentrations ranged from 40.2 to 461.5 $\mathrm{ng} / \mathrm{g}$ of feces (141.4 $\pm 12.0 \mathrm{ng} / \mathrm{g} ; \mathrm{n}=18$ individuals in 6 groups). Vigilance rates during foraging showed a positive association with mean fGC concentrations (GLMM: $\mathrm{X}^{2}{ }_{1}=18.01, \mathrm{p}=<0.001, \mathrm{n}=18$ individuals in 6 groups, for every increase of $1 \mathrm{ng}$ $\mathrm{fGCs} / \mathrm{g}$ feces, the odds of being vigilant increased by 1.008 times (Cl: 1.004, 1.011); figure 1). 


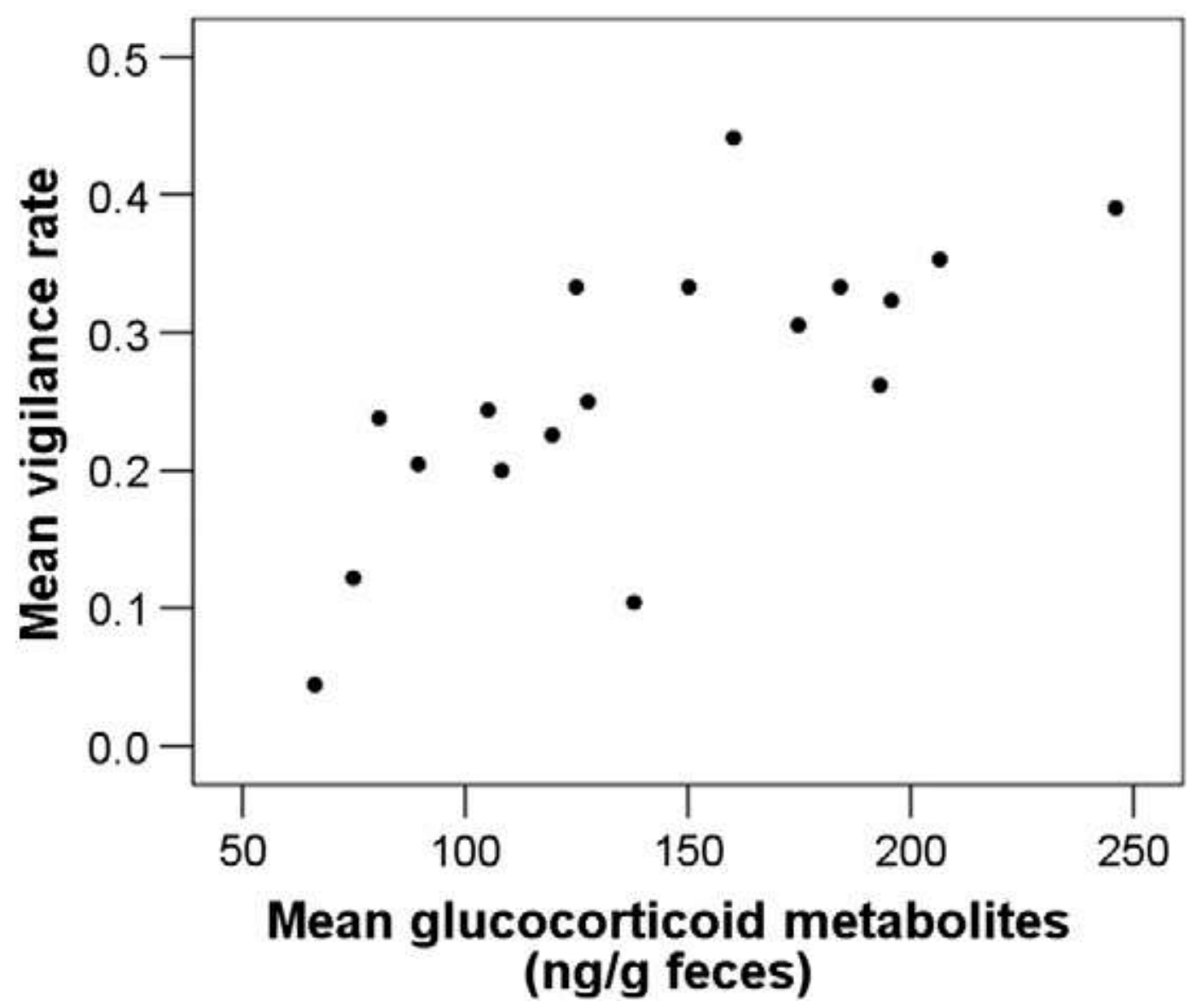

Figure 1. Relationship between individual mean fecal glucocorticoid metabolite (fGC) concentrations and mean vigilance rates observed in behavioral group scans (number of times observed vigilant/total observations for each individual). $\mathrm{N}=18$ meerkats from 6 groups.

Latency to resume foraging after responding to alarm call playbacks was not affected by mean fGC concentrations ( $\min \mathrm{fGC}=39.4 \mathrm{ng} / \mathrm{g}$ of feces, $\max \mathrm{fGC}=257.8 \mathrm{ng} / \mathrm{g}$ feces, mean \pm se $=114.5 \pm 7.4 \mathrm{ng} / \mathrm{g}$ feces, $\mathrm{MMCoxPH}: \mathrm{x}^{2}{ }_{1}=0.82, \mathrm{p}=0.366, \mathrm{n}=16$ individuals in 6 groups, 24 observations). However, animals took significantly longer to resume foraging following medium urgency alarm calls $(96.1 \pm 27.2 \mathrm{~s})$ than low urgency alarm calls $\left(12.0 \pm 3.4 \mathrm{~s}, \mathrm{x}^{2}{ }_{1}=15.17, \mathrm{p}<0.001\right.$; the odds to show first foraging behavior after low urgency alarm call playbacks were 0.065 times the latency to resume first foraging behavior after medium urgency alarm call playbacks $(\mathrm{Cl}: 0.017,0.250)$; figure $2 \mathrm{a}, 2 \mathrm{~b})$. The interaction between fGC concentrations and playback treatment was not significant $\left(\mathrm{x}^{2}{ }_{1}=0.38, \mathrm{p}=0.537\right)$. Similarly, latency to resume foraging without interruption for at least $20 \mathrm{~s}$ was not associated with fGC 
concentrations $\left(X^{2}{ }_{1}=0.46, p=0.498, n=16\right.$ individuals in 6 groups, 24 observations), whereas latency to resume foraging was significantly longer in response to medium urgency alarm call playbacks than to low urgency alarm calls (latency low urgency calls: $18.1 \pm 4.1 \mathrm{~s}, \mathrm{n}=9$; latency medium urgency calls: $110.1 \pm$ $27.6 \mathrm{~s}, \mathrm{n}=15 ; \mathrm{x}^{2}{ }_{1}=15.59, \mathrm{p}<0.001$; the odds to resume foraging without interruption after low urgency alarm playbacks were 0.074 times the latency to uninterrupted foraging after medium urgency alarm playbacks (Cl: 0.018, 0.303). Again, the interaction between fGC concentration and playback treatment was not significant $\left(X^{2}{ }_{1}=0.11, p=0.746\right)$. Furthermore, first response to alarm calls was not significantly associated with $\mathrm{fGC}$ concentrations in either level of urgency of alarm calls (Kendall's tau b: low urgency: $\mathrm{T}_{\mathrm{b}}=-0.043, \mathrm{p}=0.891, \mathrm{n}=8$ individuals in 5 groups; medium urgency: $\mathrm{T}_{\mathrm{b}}=0.047, \mathrm{p}=0.822, \mathrm{n}=15$ individuals in 6 groups); with most animals looking up standing on four legs in response to both playback call types.
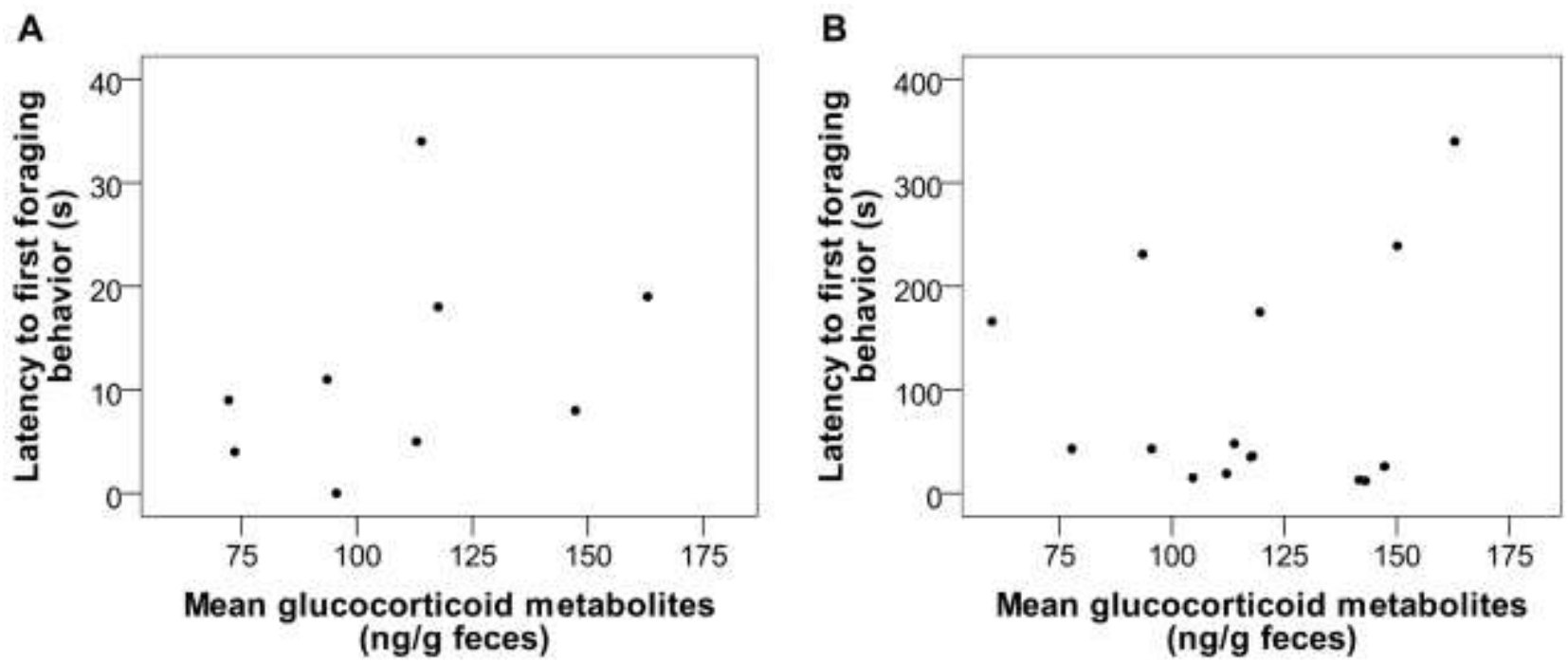

Figure 2. Relationship between individual mean fecal glucocorticoid metabolite (fGCS) concentrations and latency to display the first foraging behavior after: a) low urgency aerial alarm call playbacks $(\mathrm{N}=$ 9 meerkats from six groups; b) medium urgency aerial alarm call playbacks ( $N=15$ meerkats from six groups). 
Meerkats treated with exogenous cortisol took slightly, but significantly longer to resume foraging after an alarm call playback (mean $\pm \mathrm{se}=40.9 \pm 11.0 \mathrm{~s}, \mathrm{n}=18,36$ observations) than meerkats treated with saline control $(33.2 \pm 10.4 \mathrm{~s}, \mathrm{n}=18$; MMCoxPH: $\mathrm{X}^{2}{ }_{1}=4.39, \mathrm{p}=0.036$; cortisol administration odds 2.45 times that of control treatment $(\mathrm{Cl}: 1.10,5.48)$, figure $3 a)$. There was no significant difference between males $(23.5 \pm 4.6 \mathrm{~s}, \mathrm{n}=20)$ and females $(54.0 \pm 15.0 \mathrm{~s}, \mathrm{n}=16)$ in latency to resume foraging after the playbacks $\left(x^{2}{ }_{1}=2.44, p=0.118\right)$ and within hormonal treatments the sexes responded in similar ways to the playbacks (interaction between treatment and sex: $X^{2}{ }_{1}=0.01, p=0.930$ ). Cortisol administration did not significantly affect latency to resume foraging for at least $20 \mathrm{~s}$ without interruption (saline: $40.1 \pm 10.8, n=18,36$ observations; cortisol: $46.5 \pm 11.0, n=18, x^{2}{ }_{1}=2.56, p$ $=0.110$, figure $3 \mathrm{~b}$ ). There were no significant sex differences (males: $28.7 \pm 5.3 \mathrm{~s}, \mathrm{n}$ $=20$; females: $61.6 \pm 14.8 \mathrm{~s}, \mathrm{n}=16, \mathrm{x}^{2}{ }_{1}=2.15, \mathrm{p}=0.143$, and no significant interaction between sex and treatment $\left(x^{2}{ }_{1}=0.36, p=0.549\right)$. Cortisol/saline administration affected neither first response (Wilcoxon exact test: $Z=-0.79, p=$ $0.18, \mathrm{n}=18$ dyads, effect size $(E S)=0.13)$ nor strongest response to the alarm playbacks $(Z=-1.14, \mathrm{p}=0.28, \mathrm{n}=18$ dyads, $E S=0.19)$. 
A

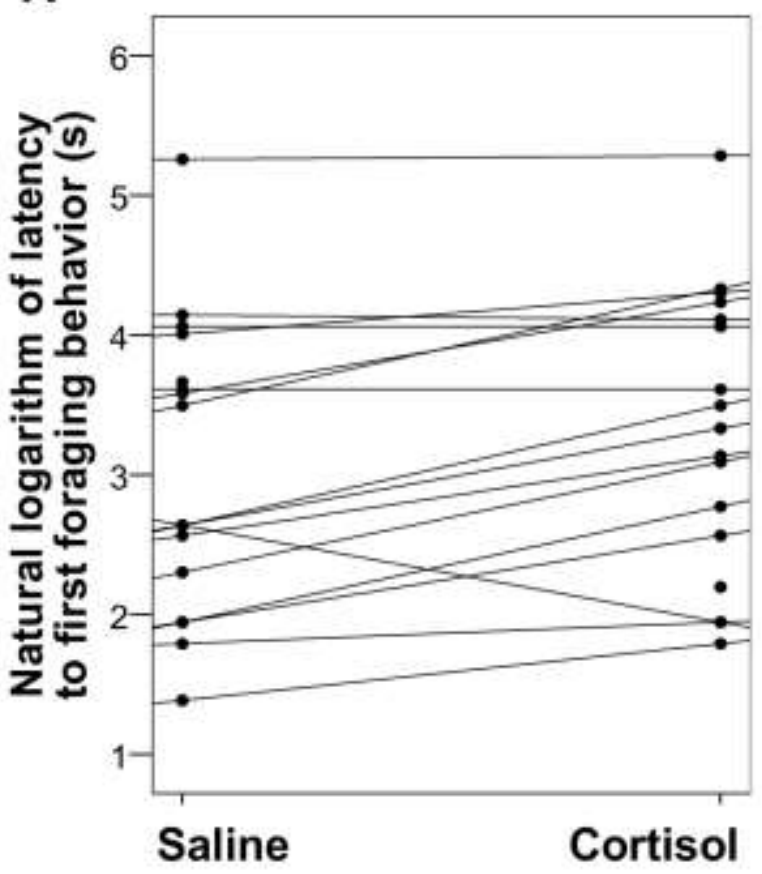

B

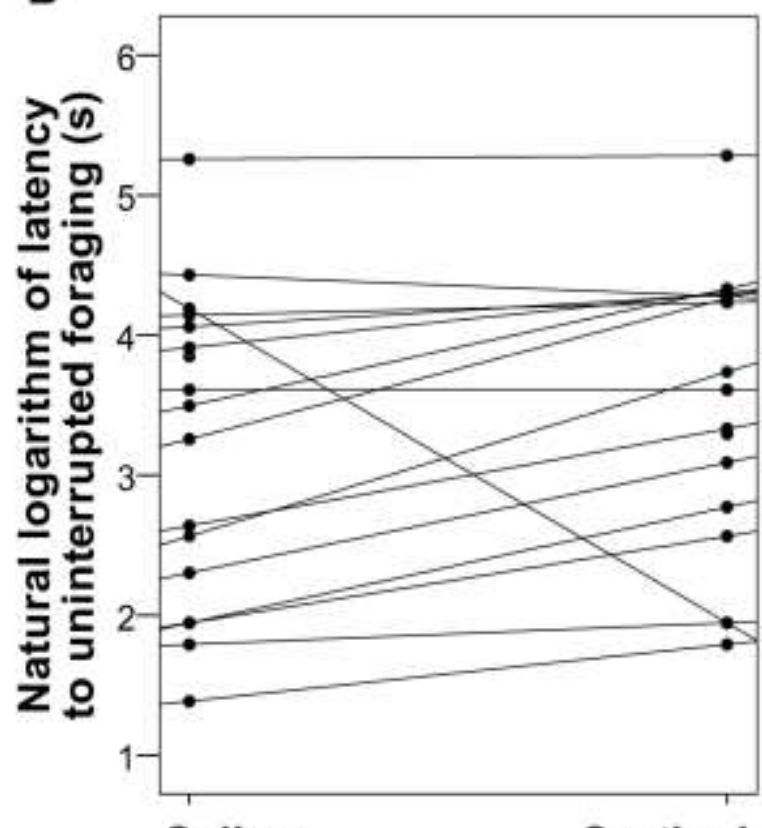

Cortisol

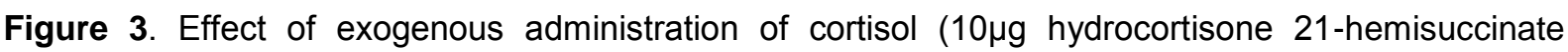
sodium salt in $0.05 \mathrm{ml}$ saline solution) and of saline solution (control, $0.05 \mathrm{ml}$ ) on: A) natural logarithm of latency (s) to show the first foraging behavior and B) natural logarithm of latency (s) to show uninterrupted foraging behavior for a minimum of $20 \mathrm{~s}$; after playbacks of medium urgency aerial alarm calls ( $N=18$ observation pairs from eight groups). Lines represent dyads of same-sex individuals tested simultaneously.

\section{Discussion}

Our experimental field study on meerkats suggests a positive association between individual mean $\mathrm{fGC}$ concentrations and mean vigilance rates during foraging, but not with immediate anti-predator responses. However, meerkats administered with exogenous cortisol took slightly longer to resume foraging after medium urgency alarm call playbacks than individuals treated with saline control. Even though these significant effects were moderate and caution is needed not to over-interpret our data, our results suggest a different association between glucocorticoid excretion and distinct anti-predator behavior, such as response to alarm calls announcing immediate threat, and preventive actions such as vigilance behavior. 
Vigilance behavior serves to detect and avoid potential dangers during activities away from shelters (Dugatkin and Godin, 1992). Whether the increase in vigilance results in an increase of GCs, or whether an increase in GCs results in an increase in vigilance is not possible to identify in our study. Increased vigilance can lead to decreased foraging activity and food intake. Thus, even though the effect of increased fGC concentrations on vigilance rates is small, it could result in decreased food intake and thus lead to fitness consequences (Hollén et al., 2008). Because environments and threats can be highly variable, the ability to adjust behavioral strategies and time-budgets according to environmental conditions is critical for survival (Mateo, 2007; Zanette et al., 2011). The ability of an animal to modulate its adrenal activity and vigilance levels depending on environmental and individual conditions can enhance threat detection and increase the likelihood of avoiding impending danger while minimizing the costs of lost foraging opportunities.

Individual differences in fGC excretion rates may arise from several, not mutually exclusive, factors. Meerkats forage in cohesive groups and therefore, within groups, individuals experience similar local environmental conditions including predation pressure.Sex, body condition, reproductive stage (Keay et al., 2006; Millspaugh and Washburn, 2004), and social context (Creel et al., 2013) are all likely to affect both adrenal activity and time required to respond to potential predators. In meerkats, dominance status (Carlson et al., 2004) and the presence of pups can influence plasma GC (Carlson et al., 2006), and reproductive stage affects mean fGC concentrations (Barrette et al., 2012). By working only with young adult subordinate individuals we aimed to minimize all the above-mentioned sources of variation. Moreover, exogenous cortisol administration did not seem to affect males and females differently, although the effect of sex on naturally occurring variability in fGC 
concentrations and on vigilance rates could not be assessed due to high eviction rates and the resulting under-representation of females in the groups during the first study period. Literature on animal personalities has shown how behavioral coping styles can affect activity patterns and GC production (Coppens et al., 2010; Koolhaas et al., 2010). For example, in great tits and in geese, reactive animals excrete higher fGC concentrations than proactive individuals, (Carere et al., 2003; Kralj-Fišer et al., 2007). Accordingly, high individual vigilance rates may arise as by-product of general higher activity levels or may result from higher GC production in response to previously experienced stressors. In our study, the behavioral responses of untreated meerkats to alarm calls did not support potential underlying differences in coping styles, as individuals with lower fGC production did not react more strongly to the playbacks. However, since we did not collect information on general activity levels, the possibility of individual differences in coping styles cannot be completely ruled out.

Based on observations of wild Belding's ground squirrels (Mateo 2007), we expected meerkats with elevated mean $\mathrm{fGC}$ concentrations to react more strongly to the alarm call playbacks. This was not the case in our study. However, meerkats administered with exogenous cortisol took, on average, $12.5 \mathrm{~s}$ longer to resume foraging in response to alarm call playbacks. During this amount of time, meerkats may find and eat prey (mean prey catch rate: one every 2 - 3 min Carlson et al., 2006) and thus, our observed increase in latency to resume foraging could result in decreased food intake (Hollén et al., 2008). Additional factors, such as distance to shelter or neighbors, as well as response of neighbors to the alarm call playbacks could also have influenced latency to resume foraging, increasing data variation and thus decreasing statistical power. Therefore, caution is needed when interpreting these 
results. Nevertheless, our results indicate that experimentally increasing plasma GCs levels may induce behavioral changes in response to predator threats, supporting previous findings from experiments on the role of GCs on meerkat cooperative behavior, where time spent foraging and association with pups were affected (Santema et al 2013). Animals treated with saline control solution did not show these behavioral changes. Thus, it is likely that cortisol manipulation have caused behavioral changes, not vice versa. Our results indicate that circulating GC concentrations can play a role in the modification of behavioral responses to alarm call playbacks, while mean fGC concentrations, averaged over one month, do not. Our observations fit with a previously proposed model suggesting that low to moderate GC concentrations primarily mediate basic regulation of metabolism and thus, potentially the amount of time dedicated to vigilance during foraging. In contrast, high concentrations of circulating GCs mediate responses to salient stressors, in our case, the response to alarm calls (Busch and Hayward, 2009; Romero et al., 2009; McEwen and Wingfield, 2003; Wingfield, 2005).

In conclusion, our data indicate that mean fGC concentrations in meerkats are positively associated with long-term anti-predator behavior, such as vigilance, whereas immediate responses to threats can be modulated by current circulating hormonal concentrations as indicated by our cortisol manipulation experiment. Together, our results suggest an association of the two different types of antipredator behavior with GCs, but that the underlying mechanisms may differ for each behavior and cannot be explained by consistent individual coping styles. Potentially, underlying mechanisms may, instead, be mediated by distinct physiological effects deriving from different concentrations of circulating active GCs and their subsequent downstream cascades, mediating stress responses versus basic regulation of 
metabolism (Busch and Hayward, 2009). Overall, our study highlights the need to investigate links between adrenal activity and anti-predatory behavior considering multiple measures of physiological change associated with metabolism regulation (e.g. heart rate, blood pressure, plasma hormone concentrations; see Sapolsky et al., 2000 for time scales of activation of different physiological components of the stress response) in parallel with measures provided by fecal hormones collected on a regular basis from known individuals (Goymann, 2012). Potential associations with immediate changes in hormonal levels would have been missed without conducting experimental hormonal manipulations. Thus, only considering the appropriate physiological measurements for specific behavioral contexts will enable us to understand whether and how physiological changes mediate behavior.

\section{Acknowledgments}

We thank T. H. Clutton-Brock and the Kalahari Research Trust for permission to work at the study site of the Kalahari Meerkat Project, as well as family $\mathrm{H}$. Kotze and neighbouring farmers for allowing us to work on their land. P. Santema, Z. Teitel and M. Fenkes greatly contributed to the cortisol manipulations and subsequent playback experiment. H. Kunc, L. Hollén, C. Müller, C. Bousquet, M. Heberlein and R. Lienert contributed to very fruitful discussions while planning this project and M. Heistermann and S. Townsend were a great help while writing this manuscript. W. Blanckenhorn and L. Gygax supported us with statistical advice. We are also grateful to the editor Kim Wallen and three anonymous reviewers for their highly valuable comments. We owe many thanks to the assistance and support of R. Sutcliffe, D. Bell, S. English, M. Finnie and all volunteers of the Meerkat Project. This study was funded by the University of Zurich and the Swiss National Science Foundation, grant no. 
31003A_13676 to IBG and MM. The long-term study site is funded by the Universities of Cambridge and Zurich.

\section{References}

Arndt, S., Turvey,C., Andreasen, N.C., 2000. Correlating and predicting psychiatric symptom ratings: Spearman's $r$ versus Kendall's tau correlation. J. Psychiat. Res. 33, 97-104. Doi: 10.1016/S0022-3956(98)90046-2.

Altmann, J., 1974. Observational study of behavior: sampling methods. Behaviour 49, 227-267. Doi: 10.1163/156853974X00534.

Amsler, V., 2009. How urgency levels in alarm calls influence the forager's response in meerkats (Suricata suricatta). MSc. thesis, University of Zurich.

Bachman, G.C., 1993. The effect of body condition on the trade-off between vigilance and foraging in Belding's ground squirrels. Anim. Behav. 46, 233-244. Doi: 10.1006/anbe.1993.1185.

Barrette, M.-F., Monfort, S.L., Festa-Bianchet, M., Clutton-Brock, T.H., Russell, A.F., 2012. Reproductive rate, not dominance status, affects fecal glucocorticoid levels in breeding female meerkats. Horm. Behav. 61, 463-471. Doi: 10.1016/j.yhbeh.2011.12.005.

Bates, D., Maechler, M., Bolker, B., 2012. Ime4: Linear mixed-effects models using S4 classes. $\mathrm{R}$ package version 0.999999-0. http://CRAN.Rproject.org/package=Ime4.

Bercovitch, F.B., Hauser, M.D., Jones, J.H., 1995. The endocrine stress-response and alarm vocalizations in Rhesus macaques. Anim. Behav. 49, 1703-1706. Doi: 10.1016/0003-3472(95)90093-4. 
Blumstein, D.T., Patton, M.L., Saltzman, W., 2006. Faecal glucocorticoid metabolites and alarm calling in free-living yellow-bellied marmots. Biol. Lett. 2, 29-32. Doi: 10.1098/rsbl.2005.0405.

Blumstein, D.T., Chi, Y.Y., 2012. Scared and less noisy: glucocorticoids are associated with alarm call entropy. Biol. Lett. 8, 189-192. Doi: 10.1098/rsbl. 2011.0832.

Boonstra, R., 2005. Equipped for life: the adaptive role of the stress axis in male mammals. J. Mammal. 86, 236-247. Doi: http://dx.doi.org/10.1644/BHE-001.1.

Busch, D.S., Hayward, L.S., 2009. Stress in a conservation context: a discussion of glucocorticoid actions and how levels change with conservation-relevant variables. Biol. Conserv. 142, 2844-2853. Doi: 10.1016/j.biocon.2009.08.013.

Carere, C., Groothuis, T.G., Moestl, E., Daan, S, Koolhaas, J.M.,2003. Fecal corticosteroids in a territorial bird selected for different personalities: daily rhythm and the response to social stress, Horm. Behav. 43, 540-548. Doi: 10.1016/S0018-506X(03)00065-5.

Carlson, A.A., Young, A.J., Russell, A.F., Bennett, N.C., McNeilly, A.S., CluttonBrock, T., 2004. Hormonal correlates of dominance in meerkats (Suricata suricatta). Horm. Behav. 46,141-150. Doi: 10.1016/j.yhbeh.2004.01.009.

Carlson, A.A., Manser, M.B., Young, A.J., Russell, A.F., Jordan, N.R., McNeilly, A.S., Clutton-Brock, T., 2006. Cortisol levels are positively associated with pupfeeding rates in male meerkats. Proc. R. Soc. Lond., B. 273, 571-577. Doi:10.1098/rspb.2005.3087 1471-2954.

Caro, T., 2005 Antipredator defenses in birds and mammals. Chicago: University of Chicago Press.

Clutton-Brock, T.H., Gaynor, D., Kansky, R., MacColl, A.D.C., Mcllrath, G., Chadwick, P., Brotherton, P.N.M., O'Riain, J.M., Manser, M., Skinner, J.D., 
1998a. Costs of cooperative behaviour in suricates (Suricata suricatta). Proc. R. Soc. Lond., B. 265, 185-190. Doi: 10.1098/rspb.1998.0281.

Clutton-Brock, T.H., Brotherton, P.N.M., Smith, R., Mcllrath, G.M., Kansky, R., Gaynor, D., O'Riain, M.J., Skinner, J.D., 1998b. Infanticide and expulsion of females in a cooperative mammal. Proc. R. Soc. Lond., B. 265, 2291-2295. Doi: 10.1098/rspb.1998.0573.

Clutton-Brock, T.H., Gaynor, D., Mcllrath, G.M., Maccoll, A.D.C., Kansky, R., Chadwick, P., Manser, M., Skinner, J.D., Brotherton, P.N.M., 1999a. Predation, group size and mortality in a cooperative mongoose, Suricata suricatta. J. Anim. Ecol. 68, 672-683. Doi: 10.1046/j.1365-2656.1999.00317.x.

Clutton-Brock, T.H., O’Rian, M.J., Brotherton, P.N.M., Gaynor, D., Kansky, R., Griffn, A.S., Manser, M., 1999b. Selfish sentinels in cooperative mammals. Science 284, 1640-1644. Doi: 10.1126/science.284.5420.1640.

Clutton-Brock, T.H., Hodge, S., Spong, G., Russell, A.F., Jordan, N.R., Bennett, N.C.,Manser, M.B., 2006. Intrasexual competition in cooperative mammals. Nature 444, 1065-1068. Doi:10.1038/7122xia.

Coppens, C.M., de Boer, S.F., Koolhaas, J.M., 2010. Coping styles and behavioural flexibility: towards underlying mechanisms. Phil. Trans. R. Soc. Lond., B. 365, 4021-4028. Doi: 10.1098/rstb.2010.0217.

Creel, S., Winnie, J.A.Jr., Christianson, D., 2009. Glucocorticoid stress hormones and the effect of predation risk on elk reproduction. P. Natl. Acad. Sci. USA, 106, 12388-12393. Doi: 10.1073/pnas.0902235106.

Creel, S., Dantzer, B., Goymann, W., Rubenstein, D.R., 2013. The ecology of stress: effects of the social environment. Funct. Ecol. 27, 66-80. Doi: 10.1111/j.13652435.2012.02029.x. 
Dallman, M.F., Strack, A.M., Akana, S.F., Bradbury, M.J., Hanson, E.S., Scribner, K.A., Smith, M., 1993. Feast and famine: critical role of glucocorticoids with insulin in daily energy flow. Front. Neuroendocrinol. 14, 303-347. Doi: 10.1006/frne.1993.1010.

Doolan, S.P., Macdonald, D.W., 1996. Diet and foraging behaviour of group-living meerkats, Suricata suricatta, in the southern Kalahari. J. Zool. 239, 697-716. Doi: 10.1111/j.1469-7998.1996.tb05472.x.

Dowle, M., Webster, K.N., Deane, E., 2012. Faecal glucocorticoid metabolite concentrations in the free-ranging bandicoots (Perameles nasuta and Isoodon obesulus) of northern Sydney. Aust. Mammal. 35, 1-7. Doi: 10.1071/AM11033.

Dugatkin, L.A., Godin, J.J., 1992. Prey approaching predators: a cost-benefit perspective. Ann. Zool. Fenn. 29, 233-252. Doi: 10.1016/s10905138(02)00157-5.

Field A., 2005. Discovering statistics using SPSS. Second Edition. SAGE Publications. London, UK.

Goymann, W., 2012. On the use of non-invasive hormone research in uncontrolled, natural environments: the problem with sex, diet, metabolic rate and the individual. Methods Ecol. Evol., 3, 757-765. Doi: 10.1111/j.2041210X.2012.00203.x.

Harvey, S., Phillips, J.G., Rees, A., Hall, T.R., 1984. Stress and adrenal function. J. Exp. Zool., 232, 633-645. Doi: 10.1002/jez.1402320332

Hollén, L.I., Manser, M.B., 2006. Ontogeny of alarm call responses in meerkats, Suricata suricatta: the roles of age, sex and nearby conspecifics. Anim. Behav., 72, 1345-1353. Doi: 10.1016/j.anbehav.2006.03.020. 
Hollén, L.I., Bell, M.B.V., Radford, A.N., 2008. Cooperative sentinel calling? Foragers gain increased biomass intake. Curr. Biol., 18, 576-579. Doi: 10.1016/j.cub.2008.02.078.

Hunter, L.T.B., Skinner, J.D., 1998. Vigilance behaviour in African ungulates: the role of predation pressure. Behaviour 135, 195-211. Doi: $10.1163 / 156853998793066320$.

Jordan, N.R., Cherry, M.I., Manser, M.B., 2007. Latrine distribution and patterns of use by wild meerkats: implications for territory and mate defence. Anim. Behav. 73, 613-622. Doi: 10.1016/j.anbehav.2006.06.010.

Keay, J.M., Singh, J., Gaunt, M.C., Kaur, T., 2006. Fecal glucocorticoids and their metabolites as indicators of stress in various mammalian species: a literature review. J. Zoo Wildl. Med. 37, 234-244. Doi: 10.1638/05-050.1.

Koolhaas, J.M., de Boer, S.F., Coppens, C.M., Buwalda, B. 2010. Neuroendocrinology of coping styles: towards understanding the biology of individual variation. Front. Neuroendocrin. 31, 307-321. Doi: 10.1016/j.yfrne.2010.04.001.

Kralj-Fišer, S., Scheiber, I.B.R., Blejec, A., Moestl, E., Kotrschal, K., 2007. Individualities in a flock of free-roaming greylag geese: behavioral and physiological consistency over time and across situations. Horm. Behav. 51, 239-248. Doi: 10.1016/j.yhbeh.2006.10.006.

Lapata, M., 2006.Automatic evaluation of information ordering. J. of Comp. Ling. 32, 471-484. doi: 10.1162/coli.2006.32.4.471.

Lima, S.L., Dill, L.M., 1990. Behavioral decisions made under the risk of predation: a review and prospectus. CAN J ZOOLOG Can. J. Zoolog. 68, 619-640. Doi: 10.1139/z90-092. 
Manser, M.B., 1999. Response of foraging group members to sentinel calls in suricates, Suricata suricatta. Proc. R. Soc. Lond. B 266, 1013-1019. Doi: 10.1098/rspb.1999.0737.

Manser, M.B., 2001. The acoustic structure of suricates' alarm calls varies with predator type and the level of response urgency. Proc. R. Soc. Lond. B., . 268, 2315-2324. Doi: 10.1098/rspb.2001.1773.

Manser, M.B., Bell, M.B., \& Fletcher, L.B., 2001. The information that receivers extract from alarm calls in suricates. Proceedings of the Royal Society of London. Series B: Biological Sciences, 268(1484), 2485-2491. Doi: 10.1098/rspb.2001.1772.

Manser, M.B., Seyfarth, R.M., Cheney, D.L., 2002. Suricate alarm calls signal predator class and urgency. Trends Cogn. Sci. 6, 55-57. Doi:10.1016/S13646613(00)01840-4.

Mateo, J.M., 2007. Ecological and hormonal associates of antipredator behavior in adult Belding's ground squirrels (Spermophilus beldingi). Behav. Ecol. Sociobiol. 62, 37-49. Doi: 10.1007/s00265-007-0436-9.

McEwen, B.S., Wingfield, J.C., 2003. The concept of allostasis in biology and biomedicine. Horm. Behav. 43, 2-15. Doi: 10.1016/S0018-506X(02)00024-7.

Millspaugh, J.J., Washburn, B.E., 2004. Use of fecal glucocorticoid metabolite measures in conservation biology research: considerations for application and interpretation. Gen. Comp. Endocrinol. 138, 189-199. Doi: 10.1016/j.ygcen.2004.07.002.

Monfort, S.L., Wasser, S.K., Mashburn, K.L., Burke, M., Brewer, B.A., Creel, S.R., 1997. Steroid metabolism and validation of noninvasive endocrine monitoring in the African Wild Dog (Lycaon pictus). Zoo Biol. 16, 533-548. Doi: 10.1002/(SICI)1098-2361(1997)16:6<533::AID-ZOO6>3.0.CO;2-B. 
Palme, R., Rettenbacher, S., Touma, C., El-Bahr, S.M., Mostl, E., 2005. Stress hormones in mammals and birds - Comparative aspects regarding metabolism, excretion, and noninvasive measurement in faecal samples. Ann. Ny. Acad. Sci. 1040, 162 - 171. Doi: 10.1196/annals.1327.021.

R Core Team, 2012. R: A language and environment for statistical computing. R Foundation for Statistical Computing, Vienna, Austria.

Romero, L.M., Reed, J.M., 2005. Collecting baseline corticosterone samples in the field: is under 3 min good enough? Comp. Biochem. Physiol. A, 140, 73-79. Doi: 10.1016/j.cbpb.2004.11.004.

Romero, L.M., Dickens, M.J., Cyr, N.E., 2009. The reactive scope model - a new model integrating homeostasis, allostasis, and stress. Horm. Behav. 55, 375389. Doi: 10.1016/j.yhbeh.2008.12.009.

Rosen, J.B., Donley, M.P., Gray, D., West, E.A., Morgan, M.A., Schulkin, J., 2008. Chronic corticosterone administration does not potentiate unconditioned freezing to the predator odor, trimethylthiazoline. Behav. Brain Res. 194, 3238. Doi: 10.1016/j.bbr.2008.06.019.

Santema, P., Teitel, Z., Manser, M., Bennett, N., Clutton-Brock, T., 2013. Effects of cortisol administration on cooperative behavior in meerkat helpers. Behav. Ecol. Doi: 10.1093/beheco/art039.

Santema, P., Clutton-Brock, T., 2013. Meerkat helpers increase sentinel behaviour and bipedal vigilance in the presence of pups. Anim Behav. 85, 655-661. doi: 10.1016/j.anbehav.2012.12.029.

Sapolsky, R.M., Romero, L.M., Munck, A.U., 2000. How do glucocorticoids influence stress responses? Integrating permissive, suppressive, stimulatory, and preparative actions. Endocr. Rev. 21, 55-89. Doi: 10.1210/er.21.1.55. 
Schibler, F., Manser, M.B., 2007. The irrelevance of individual discrimination in meerkat alarm calls. Anim. Behav. 74, 1259-1268. Doi: 10.1016/j.anbehav.2007.02.026.

Sheriff, M.J., Krebs, C.J., \& Boonstra, R. (2010). Assessing stress in animal populations: Do fecal and plasma glucocorticoids tell the same story? General and comparative endocrinology, 166(3), 614-619. Doi: 10.1016/j.ygcen.2009.12.017.

Sheriff, M.J., Dantzer, B., Delehanty, B., Palme, R., \& Boonstra, R. 2011. Measuring stress in wildlife: techniques for quantifying glucocorticoids. Oecologia, 166(4), 869-887. Doi: 10.1007/s00442-011-1943-y.

Tatalovic, M., 2008. Meerkat (Suricata suricatta) sentinel behaviour: variation in height and contribution. MPhil. thesis, University of Cambridge.

Thaker, M., Lima, S.L., Hews, D.K., 2009. Acute corticosterone elevation enhances antipredator behaviors in male tree lizard morphs. Horm. Behav. 56, 51-57. Doi: 10.1016/j.yhbeh.2009.02.009.

Therneau, T., 2012. Coxme: Mixed Effects Cox Models. R package version 2.2-3. http://CRAN.R-project.org/package=coxme

Touma, C., Palme, R., 2005. Measuring fecal glucocorticoid metabolites in mammals and birds: the importance of validation. Ann. NY. Acad. Sci. 1046, 54-74. Doi: 10.1196/annals.1343.006.

Wasser, S.K., Hunt, K.E., Brown, J.L., Cooper, K., Crockett, C.M., Bechert, U., Millspaugh, J.J., Larson, S., Monfort, S.L., 2000. A generalized fecal glucocorticoid assay for use in a diverse array of nondomestic mammalian and avian species. Gen. Comp. Endocrinol. 120, 260-275. Doi: 10.1006/gcen.2000.7557. 
Welp, T., Rushen, J., Kramer, D.L., Festa-Bianchet, M., de Passillé, A.M.B., 2004. Vigilance as a measure of fear in dairy cattle. Appl. Anim. Behav. Sci. 87, 113. Doi: 10.1016/j.applanim.2003.12.013.

Whitten, P.L., Brockman, D.K., Stavisky, R.C., 1998. Recent advances in noninvasive techniques to monitor hormone-behavior interactions. Yearb. Phys. Anthropol. 41, 1-23. Doi: 10.1002/(SICl)10968644(1998)107:27+<1::AID-AJPA2>3.0.CO;2-H.

Wingfield, J.C., 2005. The concept of allostasis: Coping with a capricious environment. J. Mammal. 86, 248-254. Doi: 10.1644/BHE-004.1.

Young, A.J., Carlson, A.A., Monfort, S.L., Russell, A.F., Bennett, N.C., Clutton-Brock, T., 2006. Stress and the suppression of subordinate reproduction in cooperatively breeding meerkats. Proc. Natl. Acad. Sci. U. S. A. 103, 1200512010. doi: $10.1073 /$ pnas. 0510038103 .

Young, A.J., Spong, G., Clutton-Brock, T., 2007. Subordinate male meerkats prospect for extra-group paternity: alternative reproductive tactics in a cooperative mammal. Proc. R. Soc. Lond., Ser. B: Biol. Sci. 274, 1603-1609. Doi: $10.1098 / \mathrm{rspb} .2007 .0316$.

Zanchi, N. E., De Siqueira, M. A., Felitti, V., Nicastro, H., Lorenzeti, F. M. \& Lancha, A. H. 2010. Glucocorticoids: Extensive Physiological Actions Modulated Through Multiple Mechanisms of Gene Regulation. J. Cell. Physiol. 224, 311315. Doi: 10.1002/jcp.22141.

Zanette, L.Y., White, A.F., Allen, M.C., Clinchy, M. 2011. Perceived predation risks reduces the number of offspring songbirds produce per year. Science, 334: 1398-1401. Doi: 10.1126/science.1210908. 